This item was submitted to Loughborough's Research Repository by the author.

Items in Figshare are protected by copyright, with all rights reserved, unless otherwise indicated.

\title{
Experimental verification of a modified Specific Anthropomorphic Mannequin (SAM) head used for SAR measurements
}

PLEASE CITE THE PUBLISHED VERSION

PUBLISHER

(c) Loughborough University

LICENCE

CC BY-NC-ND 4.0

REPOSITORY RECORD

Panagamuwa, C.J., W.G. Whittow, R.M. Edwards, and J.C. Vardaxoglou. 2019. "Experimental Verification of a Modified Specific Anthropomorphic Mannequin (SAM) Head Used for SAR Measurements". figshare.

https://hdl.handle.net/2134/2948. 
This item was submitted to Loughborough's Institutional Repository by the author and is made available under the following Creative Commons Licence conditions.

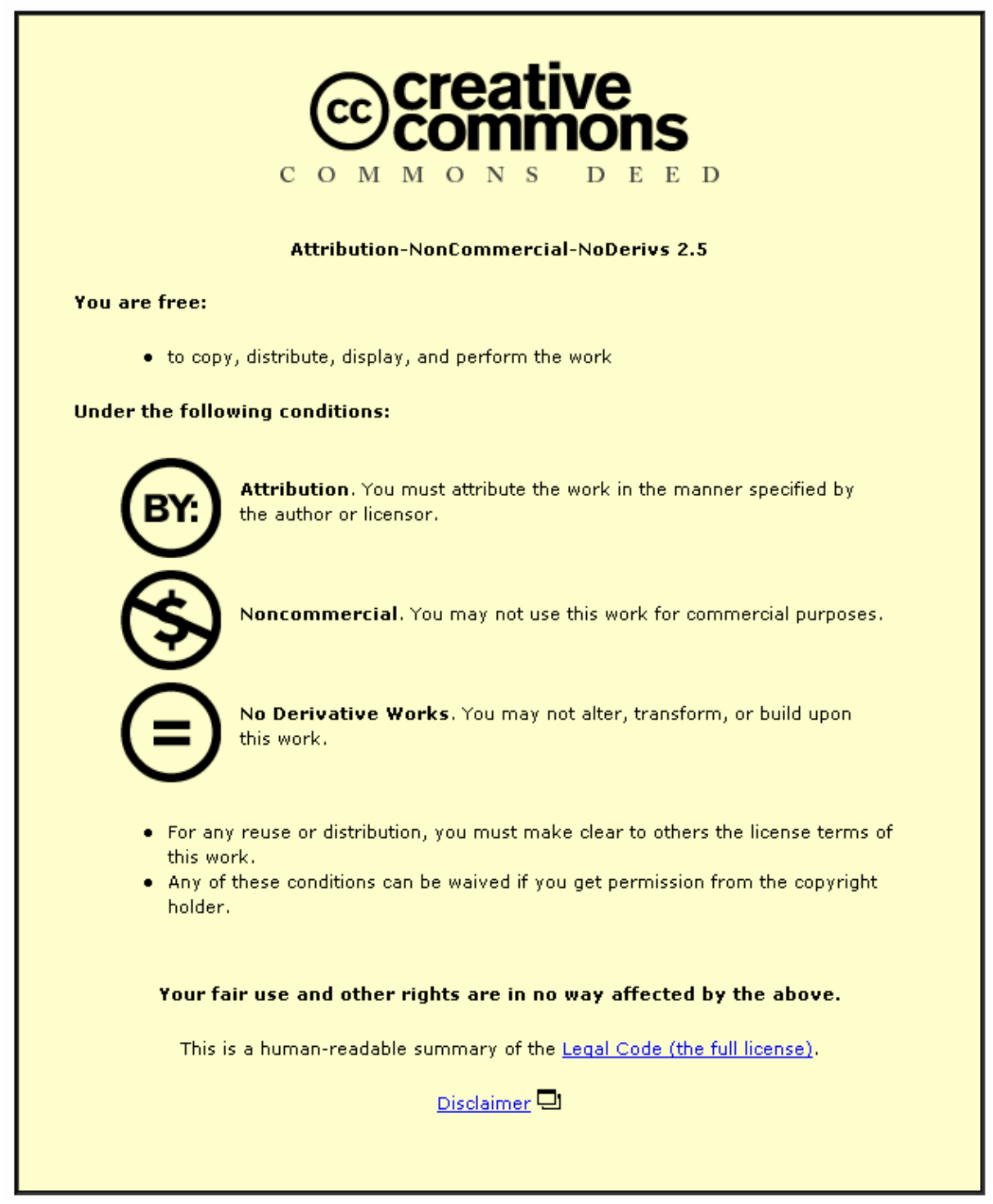

For the full text of this licence, please go to: http://creativecommons.org/licenses/by-nc-nd/2.5/ 


\title{
EXPERIMENTAL VERIFICATION OF A MODIFIED SPECIFIC ANTHROPOMORPHIC MANNEQUIN (SAM) HEAD USED FOR SAR MEASUREMENTS
}

\author{
Chinthana J. Panagamuwa ${ }^{(1)}$, William G. Whittow ${ }^{(2)}$, R. M. Edwards ${ }^{(3)}$ and John C. \\ Vardaxoglou ${ }^{(4)}$
}

\author{
Department of Electronic \& Electrical Engineering, Loughborough University, \\ Loughborough, Leicestershire, LE11 3TU, UK \\ ${ }^{(1)}$ Email: c.j.panagamuwa@lboro.ac.uk \\ ${ }^{(2)}$ Email: w.g.whittow@lboro.ac.uk \\ (3)Email: r.m.edwards@lboro.ac.uk \\ (4)Email: j.c.vardaxoglou@lboro.ac.uk
}

\begin{abstract}
This paper investigates a method of facilitating Specific Absorption Rate (SAR) measurements in the head when using a $1800 \mathrm{MHz}$ radiation source placed in front of the face. A Specific Anthropomorphic Mannequin (SAM) head phantom is modified by removing the rear most part to enable fully-automated scanning of the face region by a DASY4 electric-field probe. Prior to the modification, simulations were carried out in FDTD to establish the optimum area to be removed. This paper compares predicted local SAR values behind the face with actual measurements carried out using the new modified SAM phantom head. Measurements show good agreement with simulations, indicating that the modified SAM head is suitable for SAR measurements when the source is placed in front of the face.
\end{abstract}

\section{Introduction}

The number of Personal Data Assistants incorporating Mobile Communications Equipment (PDAMCE) in use today has seen a rapid increase due to advances in technology, introduction of $3 \mathrm{G}$ and user demand for higher functionality. Such devices have comparatively large displays and are intended for use in front of the face. In these systems the RF source is predominantly sited toward the front of the head rather than the side of the head which is the case for typical mobile phones. As a consequence the eyes which have been found to be a sensitive organ when considered with RF energy, may receive rather more direct illumination. The authors [1][2] have shown in the past that certain metallic spectacles can increase the SAR in the eyes. Other Finite-Difference Time-Domain (FDTD) studies have also examined the SAR in the eyes when radiated from in front of the face [3] - [5]. Measurements were carried out by Balzano [6] and Cleveland [7] using human skulls filled with brain simulating materials. While there are many FDTD based studies, measurements from in front of the face using the recently introduced SAM head phantom standard [8] are less common.

SAR measurements for standards purposes are generally done using an automated E-field scanning system such as DASY4 [9]. The DASY4 uses a right and left half head shell filled with brain tissue simulating liquid. During irradiation by a mobile phone a robot positioned E-field probe is dipped into the liquid inside the head and using the known properties and the measured total electric field the SAR can be calculated. The geometry of the DASY4 system although well suited to for the measurement of devices held to the ear is less amenable to measurements of devices held to the face. Previously, the authors proposed a method to integrate a modified SAM head phantom into the DASY4 to facilitate SAR measurements inside the face [10]. That study used FDTD to look at the effects of removing increasingly larger sections from the back of the head on local SAR along three different cuts into the head; namely the nose, eye and eyebrow. That paper also looked at the effects on $1 \mathrm{~g}$ and $10 \mathrm{~g}$ SAR. Herein the theoretical studies of [10] are validated by SAR measurements. The increase in facility for measurements is also evaluated.

\section{Rear-Opened SAM Head Phantom}

The SAM head phantom used in this study provided by SPEAG is normally intended for radiation pattern and efficiency measurements. It is constructed by binding two opposite halves of the head together and has a continuous seam that runs along the join. There is a hole at the top of the head, through which the phantom is filled with brain simulating liquid. In order to gain access to the face region, the back of the head was removed. A previous study [10] investigated the size of the section that can be safely removed. As the depth of the 
removed section becomes larger, the depth of the brain simulating liquid inside the head becomes insufficient for accurate SAR measurements. The RF reflections off the surface of the liquid start to interfere with the E-field measurements behind the face and so cause errors in the SAR calculations. However, removing larger sections improves access to the face region, specially since the E-field probe tip has to approach the phantom surface within $30^{\circ}$ of the normal in order to take an accurate measurement. The previous FDTD investigation using a $2 \mathrm{~mm}$ resolution model of the SAM head phantom showed that $100 \mathrm{~mm}$ could be safely removed from the back of the head without causing any noticeable change to the local SAR along three cuts in to the head (nose, eye and eye brow) [10]. However, this would remove part of the ears and disrupt the hole at the top of the head, thus reducing its mechanical stability. Therefore, a compromise was reached and a section $6 \mathrm{~mm}$ deep was removed from the back of the head, leaving both ears intact. Figure 1 shows photographs of the modified SAM head from three different angles.

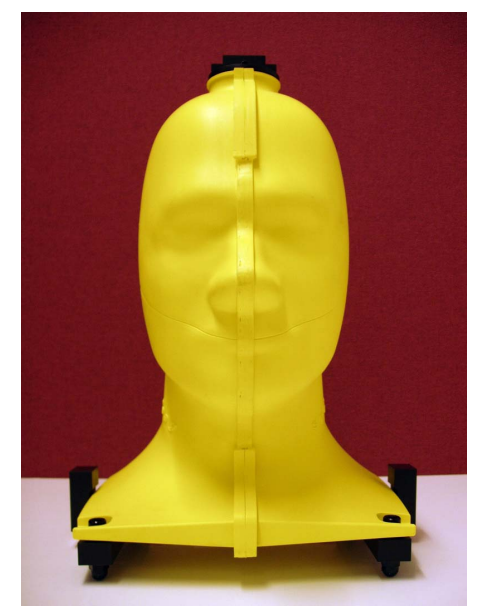

(a)

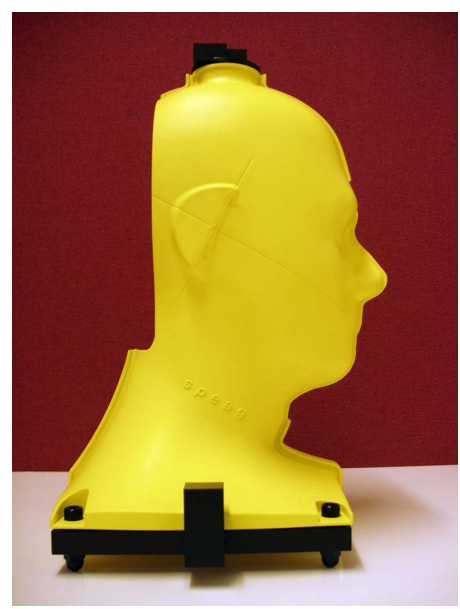

(b)

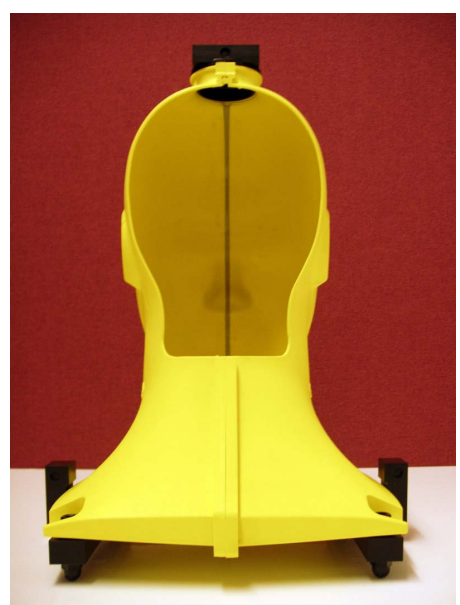

(c)

Figure 1 - Modified SAM head phantom (a) front view, (b) side view, (c) back view

The joining seam on the SAM head is clearly visible in Figure 1(a). This seam is essential to maintain structural stability and prevent leakages and would normally protrude from the surface by about $7 \mathrm{~mm}$. However, in the area spanning the front of the face, the seam height has been reduced (see Figure 1(a) and (b)). On the inside of the phantom, there is also a thin channel that runs along the inside of the seam (see Figure 1(c)). This has been filled in using a water resistant plastic filler. The effects on SAR due to this modified seam and filled in channel were investigated through simulation and measurement.

Figure 2 shows the modified SAM head integrated into the DASY4 kit. The existing twin phantom is removed and the SAM head is fixed horizontally using two plastic fixtures. This arrangement ensures there are no interfering structures below the face region and allows easy placement of an RF source (mobile phone, PDA, dipole antenna etc.)

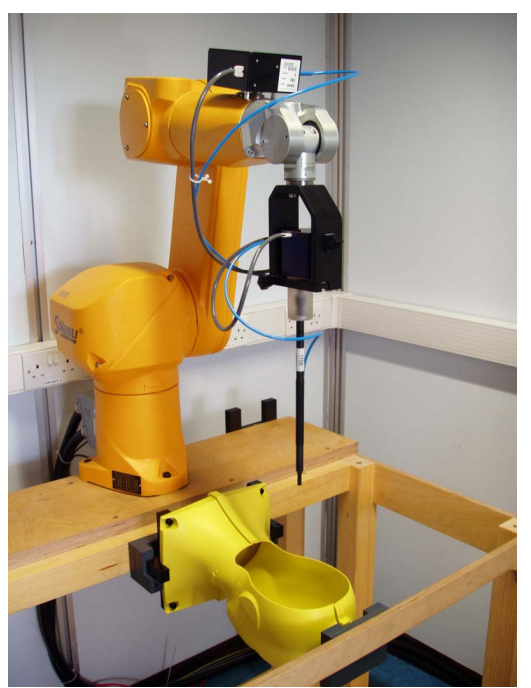

Figure 2 - Modified SAM head phantom integrated into DASY4 


\section{Measured and Simulated Results}

In order to validate the simulated results in [10], the DASY4 kit was used to measure the local SAR along three different cuts into the head, namely through the tip of the nose, the eye and eyebrow at $1800 \mathrm{MHz}$. The nose and eyebrow are of particular interest because of the possibility of studying the effects of metallic jewellery commonly found at these locations. Metallic rims of spectacles have been known to cause changes in SAR in the eye [1] and so this location is also of interest.

An in-house FDTD solver [1] was used to calculate the SAR along the three different cuts. The IEEE 1529a-2005 SAM head phantom was descretised and imported into the code with a resolution of $2 \mathrm{~mm}$. The $2 \mathrm{~mm}$ thick shell was assigned properties of $\varepsilon_{\mathrm{r}}=3.5$ and $\sigma=0 \mathrm{~S} / \mathrm{m}$. The tissue inside was assigned $\varepsilon_{\mathrm{r}}=$ $40.48, \sigma=1.37 \mathrm{~S} / \mathrm{m}$ and $\rho=1000 \mathrm{~kg} / \mathrm{m}^{3}$.

For the measurements, a $72.5 \mathrm{~mm}$ long dipole source was placed horizontally below the head phantom along the axis of the head. See Figure 3 for location of cuts and dipole orientation. The perpendicular distance from the lower most point of the nose to the dipole was fixed at $80 \mathrm{~mm}$. The dipole was then offset by $44 \mathrm{~mm}$ towards the top of the head so that the centre of the dipole was roughly in line with the eyes. The dipole was fed with a CW source at $1800 \mathrm{MHz}$ and all measurements were normalised to $1 \mathrm{~W}$. All alignments were carried out using lasers, plumb lines and spirit levels for maximum accuracy.

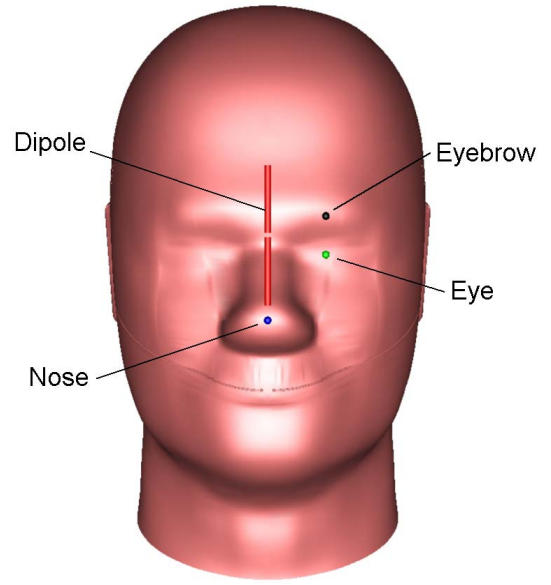

Figure 3 - Position of the three cuts into the head and the orientation of the dipole

SAR readings were taken as the E-field probe retracted along the vertical axis starting from the surface of the phantom. Graphs comparing the local SAR along the three cuts between measurements and simulations are shown in Figure 4. All three measured cuts show good agreement with the simulated results. The cut through the nose shows that the local SAR has a maximum $20 \mathrm{~mm}$ into the nose. As seen in [10], the energy seems to be reflected off the sides of the nose causing the peak SAR to occur $20 \mathrm{~mm}$ inside the nose and not at the surface.
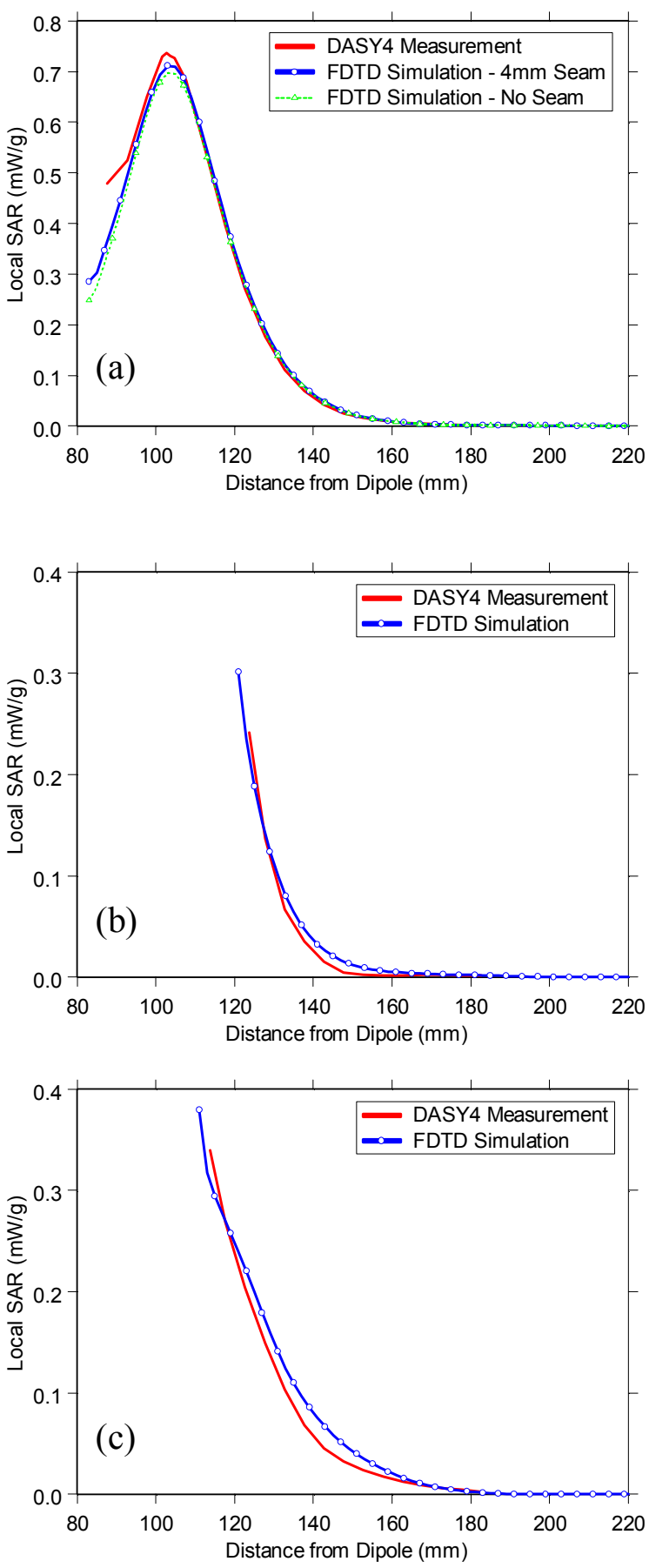

Figure 4 - Measured and Simulated Local SAR along cuts in to the head at the (a) nose, (b) eye and (c) eyebrow

In the nose cut, the measurement closest to the surface is unreliable because of the proximity of the E-field probe tip to the inner walls of the nose. The electric fields around the probe tip are perturbed when in proximity to the 
phantom shell. When the probe is inside the nose tip, the curved shape of the nose places the tip even closer to the shell, thus increasing the error in the E-field reading.

As can be seen in Figure 1(a) and (b), there is a seam that runs along the front of the face. The normal seam is $16 \mathrm{~mm}$ wide and $7 \mathrm{~mm}$ deep. However, the height and width of the seam have been reduced in most areas of the face to a width of $10 \mathrm{~mm}$ and a thickness of $3 \mathrm{~mm}$. The FDTD simulation models a seam of $10 \mathrm{~mm} \times 4 \mathrm{~mm}$ along the full length of the face. Simulation results with and without the $4 \mathrm{~mm}$ seam are shown in Figure 4(a). Introducing a seam increases the peak SAR by only $2 \%$ with very little noticeable difference elsewhere.

Figure 1(c) shows a channel that runs along the inside of the phantom head behind the outer seam. The channel is $3.5 \mathrm{~mm}$ wide and $2 \mathrm{~mm}$ deep and is a result of the joining of two half heads. It has been filled in with a water resistant sealant. As the dielectric properties of the sealant are unknown, it is not possible to simulate it in the FDTD software. Instead, the simulation models the area as a continuous shell and so does not include a channel. This may also contribute to the small discrepancy seen between simulation and measurement in Figure 4(a).

The accessibility of the inside face by the E-field probe was evaluated by attempting detailed automated area scans with the probe. The results showed good probe coverage with only the steep parts of the nose inaccessible.

\section{Conclusions}

Our opinion is that the new modified SAM head phantom may be reliably used for DASY4 SAR measurements in the head when using a RF source in front of the face at $1800 \mathrm{MHz}$. The local SAR measured into the head at three points of interest agreed well with simulated FDTD results. The seam that runs along the front of the face has minimal effect on the local SAR. Future research will include a sensitivity study and a series of experiments at other popular mobile communications frequencies.

\section{References}

[1] Whittow, W.G. and R.M. Edwards, A study of changes to specific absorption rates in the human eye close to perfectly conducting spectacles within the radio frequency range 1.5 to $3.0 \mathrm{GHz}$. IEEE Trans. Antennas and Propagation, 2004. 52(12): p. 3207-3212

[2] Whittow, W.G. and R.M. Edwards, Applications of a genetic algorithm for identification of maxima in specific absorption rates in the human eye close to perfectly conducting spectacles. IEE Proceedings Science, Measurement \& Technology, 2005. 152(3): p. 89-96.

[3] Taflove, A. and M.E. Brodwin, Computation of the electromagnetic fields and induced temperatures within a model of the microwave-irradiated human eye. IEEE Trans. Microwave Theory Technology, 1975. 23(11): p. 888-896.

[4] Dimbylow, P.J. and O.P. Gandhi, Finite-difference time-domain calculations of SAR in a realistic heterogeneous model of the head for plane-wave exposure from $600 \mathrm{MHz}$ to $3 \mathrm{GHz}$. Physics in Medicine and Biology, 1991. 36(8): p. 1075-1089.

[5] Hirata, A., G. Ushio, and T. Shiozawa, Formation of hot spots in the human eye for plane wave exposure. Microwave Conference, 1999 Asia Pacific, 1999. 2: p. 477-480.

[6] Balzano, Q.O. Garay and F. R. Steel, Energy deposition in simulated human operators of $800-\mathrm{MHz}$ portable transmitters, IEEE Transactions on Vehicular Technology, 1978. 27(4): p. 174-182.

[7] Cleveland, R.F. and T.W. Athey, Specific absorption rate (SAR) in models of the human head exposed to hand-held UHF portable radios. Bioelectromagnetics, 1989. 10: p. 173-186.

[8] IEEE Recommended Practice for Determining the Peak Spatial-Average Specific Absorption Rate (SAR) in the Human Head from Wireless Communications Devices: Measurement Techniques, IEEE Standard 1528-2003, 2003.

[9] Schmid \& Partner Engineering AG, DASY4 Manual v4.1, March 2003

[10] C. J. Panagamuwa, W. Whittow, R. Edwards, J. C. Vardaxoglou and P. McEvoy, "A study of the validation of RF energy Specific Absorption Rates for simulations of anatomically correct head FDTD simulations and truncated DASY4 standard equipment measurements", European Conference on Antennas and Propagation, Nice, France, November 2006. 